\title{
A Novel Method Based on RFID Rotating Antenna for Indoor Localization
}

\author{
Yue Zhang ${ }^{1, \text { a }}$, Run Zhao ${ }^{2, \mathrm{~b}}$, Qian Zhang ${ }^{1, \mathrm{c}}$ and Dong Wang ${ }^{1, \mathrm{~d}}$ \\ ${ }^{1}$ School of Software, Shanghai Jiao Tong University, Shanghai 200240, China; \\ ${ }^{2}$ Computer Science Department, Shanghai Jiao Tong University, Shanghai 200240, China.
}

Keywords: RFID, localization, SAR.

\begin{abstract}
With the rapid growth IoT (Internet of Things), indoor positioning system has attracted significant interest, especially in complex environment. UHF RFID system has the potential to estimate the accurate location of indoor objects due to the long reading range and multi-tag identification capability. This paper introduces indoor localization system using a novel concept for robot based on a rotating UHF RFID antenna. A rotating platform is deployed on robot to rotate RFID antenna. It leverages Synthetic Aperture Radar (SAR) approach to locate robot position in real time when robot trajectory is indistinct or even unknown. The system is proved to be a promising solution with an average error with cm-level.
\end{abstract}

\section{Introduction}

With the development of technology, we are going through the Industrie 4.0, considered as the 4th Industrial Revolution. Since CPS (Cyber Physical System) are introduced into manufacturing, manufacturing factory became smart, awareness and autonomy[1], which are features for Industrie 4.0. A significant issue is raised that how to locate transport robots in factory. GPS is widely used in outdoor localization, but it is not suitable for indoor moving object, which is the key to robots scheduling in industry.

RFID (radio frequency identification) technology is widely applied in industries due to the capability of wireless identification and tracking. Compared with other identification technology, RFID system has the advantage of non-contact communication, long reading range, low cost and easy-deployment. RFID tags are usually in small size and can be easily attached to the items[2].

It was shown by Klausing[3] that it is possible to create a high-resolution 360 2D image using a SAR with rotating antennas (ROSAR) in radar system. RFID and radar are both wireless technology and work on similar theory. Both [4] and [5] exploit method based on rotating antenna to obtain position of target object. Yet, this approach is complex, the RF transmitters and receivers manufacturing cost are not as lower as available RFID readers and tags, and it cannot be taken into the practical indoor localization system. SAR (Synthetic Aperture Radar) and ISAR (Inverse Synthetic Aperture Radar) could also be exploited in RFID localization system. PinIt[6] and Tagoram[7] utilize ISAR to improve accuracy, and promotes the location accuracy, obtaining high-resolution. However, none of these localization method based on RFID gather radio signal data while reader antenna rotating.

In this paper, a RFID localization and tracing system based on RFID phase and SAR holography is proposed. We utilize a novel robot carrying rotating platform deployed with a RFID reader antenna. In order to track accurately, we build a SAR hologram based on a probabilistic model with RFID phase values, and the average tracking accuracy could be improved.

\section{System Setup and Holographic Reconstruction Algorithm}

The RFID localization system comprises a rotating RFID reader antenna attached to the top of a mobile robot concept and RFID tags deployed in indoor. The trajectory of antenna is distributed into $M$ measurement points spatially, which compose a synthetic aperture. 


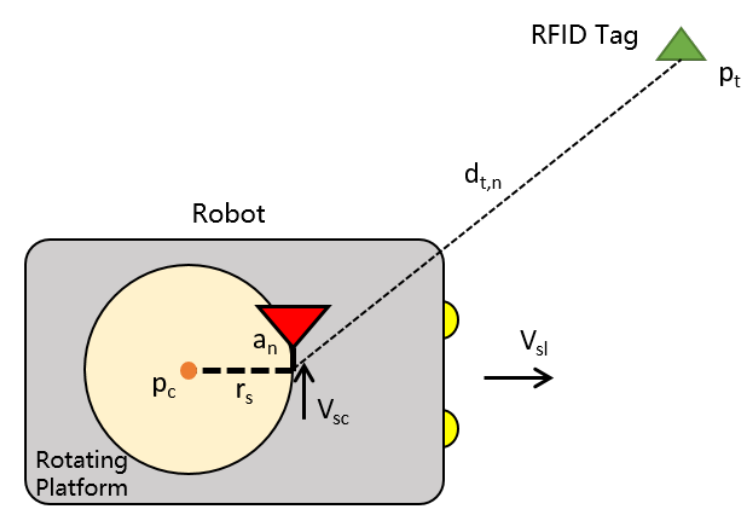

Fig. 1 Radar scanner for robot navigation.

Fig. 1 shows how the structure of our mobile robot with rotating platform work. The radius of antenna's motion is defined as $r_{s}$, and the center of rotation $\mathbf{p}_{\mathbf{c}}$ is also the origin of $X Y$ plane in coordinate system, which can be defined as $\left(\mathrm{x}=0, \mathrm{y}=0, \mathrm{z}=\mathrm{z}_{0}\right)$. The position of antenna is assumed as $\mathbf{a}_{n}=\left(\mathrm{x}_{\mathrm{n}}, \mathrm{y}_{\mathrm{n}}, \mathrm{z}_{0}\right)$, corresponding to the $n$th aperture. Antenna doing constant rotation with the velocity $\mathbf{v}_{s c}$, while the robot move with velocity $\mathbf{v}_{s l}$. A number of UHF RFID tags locate in this spatial is assumed at point $\mathbf{p}_{t}=\left(\mathrm{x}_{\mathrm{t}}, \mathrm{y}_{\mathrm{t}}, \mathrm{z}_{0}\right)$. For some of these tags, their positions are known, but others are unknown. We can exploit these know position tag to adjust trajectory of robot due to velocity sensor that is not precise. There are $\mathrm{M}$ different measurement positions defined as $\mathbf{a}_{n}(n=0 \ldots M-1)$ on the trajectory of antenna caused by the system movement.

The distance between RFID antenna at the nth aperture points $\mathbf{a}_{n}$ and UHF RFID tag at $\mathbf{p}_{t}$ can be formulated in (1) from Fig. 1.

$$
d_{t, n}=\left|\mathbf{p}_{t}-\mathbf{a}_{n}\right|=\sqrt{\left(x_{t}-x_{n}\right)^{2}+\left(y_{t}-y_{n}\right)^{2}+\left(z_{t}-z_{0}\right)^{2}}
$$

The communication between RFID reader and the UHF RFID tag is backscatter radio link. If we assume round trip time of flight between reader antenna and tag is $\tau$, then the transmitted signal of RFID reader is given as

$$
S(t)=A_{t} e^{j\left(\omega_{c}(t-\tau)+\varphi_{0}\right)}
$$

where $A_{n}$ is the amplitude of the signal modulated by the tag, $\omega_{c}$ is the center frequency, and $\varphi_{0}$ is RFID phase offset caused by additional phase rotation, introduced by reader's transmitter, tag's reflection characteristic[7], [8]. $S(t)$ is Theoretical received signal.

For synthetic aperture method, every point is correlated with measured received signal, which is the real one. We assume $E\left(t, \mathbf{a}_{n}, \mathbf{p}_{r}\right)$ to be the real received signal, the function is given as

$$
E(t)=A_{t} e^{j\left(\omega_{c}\left(t-\tau^{\prime}\right)+\varphi_{0}\right)} .
$$

$S(t)$ is corresponding hypothetical signal with $E(t)$. The real received signal will reach a large value when the measured received signal is similar to the theoretical signal, which means correlation coefficient $C\left(d_{r, n}\right)$ of $S(t)$ and $E(t)$ will have a high amplitude. $C\left(d_{r, n}\right)$ can be written as [9]

$$
C\left(d_{r, n}\right)=\int_{-\infty}^{\infty} E(t) \cdot S_{n}^{*}(t) d t=A_{t}^{2} e^{j\left(\omega_{c}\left(\tau-\tau^{\prime}\right)\right)}=A_{t}^{2} e^{j\left(\frac{2 \omega_{c}}{c}\left(d-d_{r, n}\right)\right)}
$$

where $d_{r, n}$ represents antenna and tag are at position $\mathbf{a}_{n}$ and $\mathbf{p}_{r}$ respectively.

Signal is not continuously measured and the aperture trajectory is distributed into $M$ measurement points spatially. we sum up $C\left(d_{r, n}\right)$ along trajectory, the likelihood function $\mathfrak{R}\left(\mathbf{p}_{r}\right)$ is inferred as (5). $\mathfrak{R}\left(\mathbf{p}_{r}\right)$ will reach maximum when $d_{r, n}=d_{0, n}$ if there is a tag at position $\mathbf{p}_{0}$, result from the phase 
superimposing for $\mathbf{p}_{0}$. Signals from hypothetical tags in the false position and noise components will be summed up incoherently, which lead to a small value of $\mathfrak{R}\left(\mathbf{p}_{r}\right)$.

$$
\mathfrak{R}\left(\mathbf{p}_{r}\right)=\left|\sum_{n=0}^{M-1} C\left(d_{r, n}\right)\right|=\left|\sum_{n=0}^{M-1} A_{t}^{2} e^{j\left(\frac{2 \omega_{c}}{c}\left(d-d_{r, n}\right)\right)}\right| .
$$

\section{Simulation Results}

Before measurement test, the simulation test is essential for demonstration in advance. In the simulation, a scenario is defined as below: the rotation rate of rotating platform is $9 \mathrm{r} / \mathrm{min}$, the rotation radius of antenna $r_{s}$ is $0.3 \mathrm{~m}$, and there is a tag located at $\mathbf{p}_{0}(2 \mathrm{~m}, 2 \mathrm{~m})$.

If the robot is stationary $\left(\mathbf{v}_{s l}=0\right.$ ), the RFID reader antenna is just rotating. So the trajectory of synthetic aperture is a circle. The phase measurement simulation has been done for a frequency $f=920 \mathrm{MHz}$. Here the residual velocity is not considered temporarily, it is an ideal situation. Hence, holographic image is calculated with formula (5). The 2-D SAR holographic image for tag relative location to antenna is depicted in Fig. 3(a). The maximum pixel point is $(2.06 \mathrm{~m}, 2.05 \mathrm{~m})$. If this tag is position known, the position of antenna can be determined. We suppose that robot is doing linear motion along $X$ axis. Fig. 3(b) and Fig. 2(c) show the simulation results when velocity $\mathbf{v}_{s l}$ is $0.5 \mathrm{~m} / \mathrm{s}$ and $1 \mathrm{~m} / \mathrm{s}$. The maximum pixel point is $(2.01 \mathrm{~m}, 2.02 \mathrm{~m})$ and $(2 \mathrm{~m}, 1.99 \mathrm{~m})$ in respective holographic image, and both of them are close to point $\mathbf{p}_{0}$.

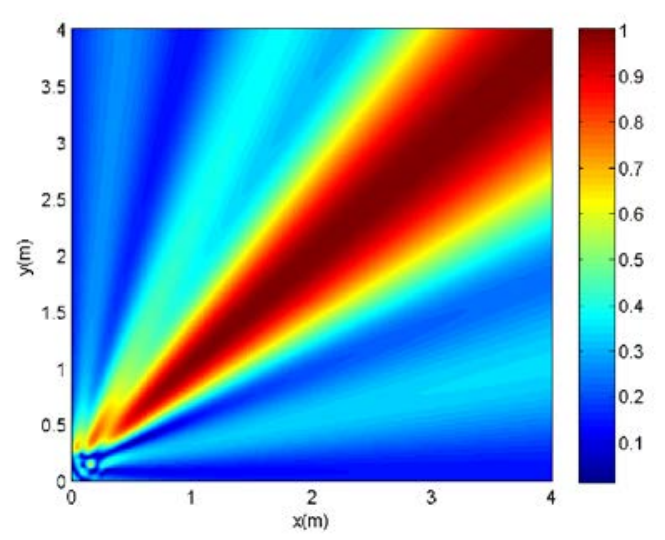

(a) $\mathbf{v}_{s l}=0$

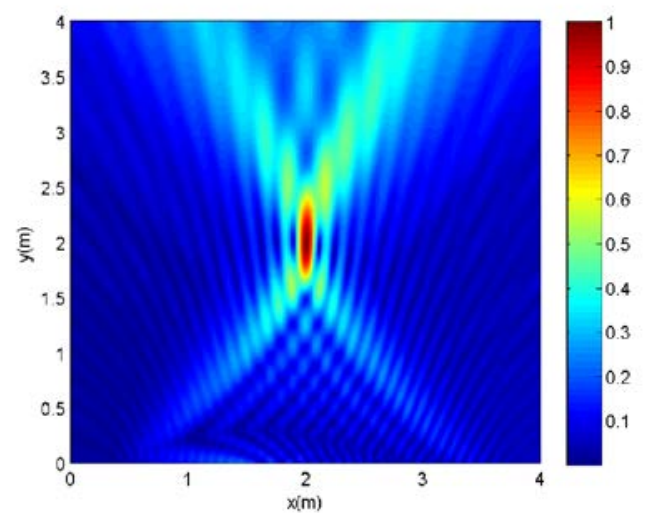

(c) $\mathbf{v}_{s l}=1 \mathrm{~m} / \mathrm{s}$

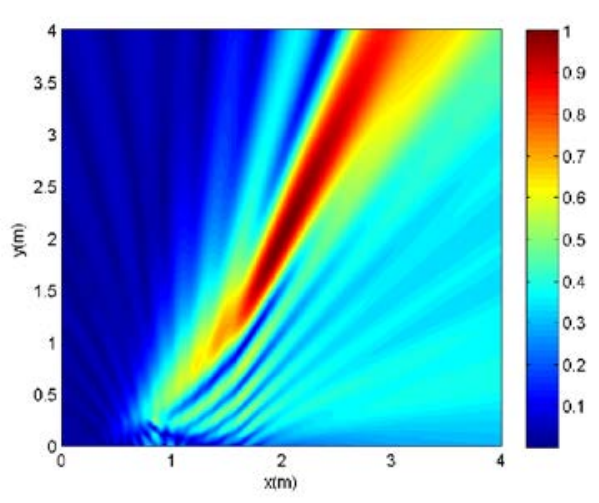

(b) $\mathbf{v}_{s l}=0.5 \mathrm{~m} / \mathrm{s}$

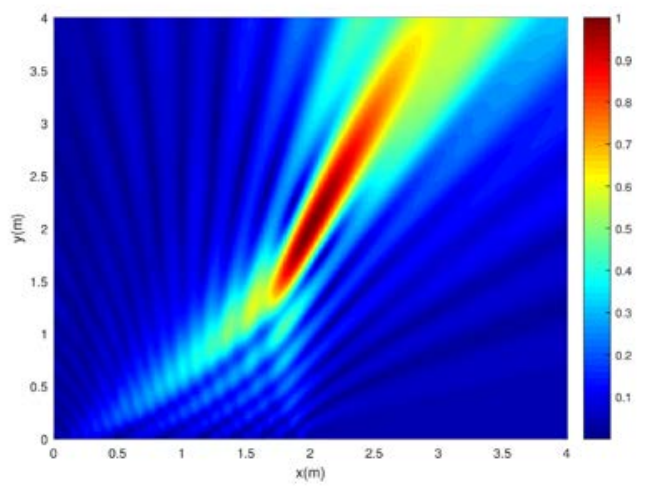

(d) $\mathbf{v}_{s c}=0 \quad \mathbf{v}_{s l}=1 \mathrm{~m} / \mathrm{s}$.

Fig. 3 The SAR hologram for simulation experiment

Comparing Fig. 3(c) and Fig. 3(d), it is inferred that rotation contributes to the accuracy on $Y$ axis. Relative movement on $Y$ axis due to antenna's rotation between antenna and tag actually promotes the image resolution on $Y$ axis. 
It comes to a conclusion that movement and velocity of robot can make an influence on location accuracy. Longer trajectory will lead to higher location accuracy. It's significant to make a trade-off decision between robot's velocity and synthetic aperture time.

\section{Prototype System}

Impinj Speedway modeled R420 reader and Alien 9740 RFID tags is chosen for signal acquisition. This model of reader supports to obtain RFID phase value. After the experimental simulation, parameters of this prototype system is in accord with the simulation scenario, and velocity of robot is set as $\mathbf{v}_{s l}=0.5 \mathrm{~m} / \mathrm{s}$, which the result of comprehensive consideration of location accuracy and synthetic aperture time. The frequency $f$ of RFID reader is also $920 \mathrm{MHz}$.

In this experiment, RFID tags' position is already known, but the start point of robot is unknown in complex environment. The trajectory of robot is indistinct. According to our approach, the relative location between RFID tags and antenna can be calculated using the algorithm above.

\section{Summary}

In this paper, we promote a novel method based on RFID rotating antenna for indoor robot location. According to our algorithm, the relative location between robot and RFID tags can be obtain, and the robot location can be inferred if the tag location is known. Synthetic Aperture Radar is introduced into RFID signal processing to build holographic image. The simulation and experiment show achievement for indoor localization with cm-level accuracy.

\section{References}

[1]H. Kagermann, W. Wahlster, and J. Helbig, Recommendations for Implementing the Strategic Initiative INDUSTRIE 4.0: Securing the Future of German Manufacturing Industry; Final Report of the Industrie 4.0 Working Group, no. April. Forschungsunion, 2013.

[2]R. Miesen, R. Ebelt, F. Kirsch, T. Scha04Fer, G. Li, H. Wang, and M. Vossiek, 'Where is the Tag?’, IEEE Microw. Mag., vol. 12, no. 7, pp. S49 - S63, 2011.

[3]H. Klausing, 'Feasibility of a Synthetic Aperture Radar with Rotating Antennas (ROSAR)', 19th Eur. Microw. Conf. 1989, pp. 287-299, 1989.

[4]A. Ledeczi, P. Volgyesi, J. Sallai, and R. Thibodeaux, 'A novel RF ranging method', in Intelligent Solutions in Embedded Systems, 2008 International Workshop on, 2008, pp. 1-12.

[5]F. Ali, G. Bauer, and M. Vossiek, 'A Rotating Synthetic Aperture Radar Imaging Concept for Robot Navigation', IEEE Trans. Microw. Theory Tech., vol. 62, no. 7, pp. 1545-1553, 2014.

[6]J. Wang and D. Katabi, 'Dude, where's my card?: RFID positioning that works with multipath and non-line of sight', in ACM SIGCOMM Computer Communication Review, 2013, vol. 43, no. 4, pp. 51-62.

[7]L. Yang, Y. Chen, X. Li, C. Xiao, M. Li, and Y. Liu, 'Tagoram : Real-Time Tracking of Mobile RFID Tags to High Precision Using COTS Devices Categories and Subject Descriptors', in MobiCom, 2014.

[8]S. Särkkä, V. V Viikari, M. Huusko, and K. Jaakkola, 'Phase-based UHF RFID tracking with nonlinear Kalman filtering and smoothing’, Sensors Journal, IEEE, vol. 12, no. 5, pp. 904-910, 2012.

[9]A. Parr, R. Miesen, and M. Vossiek, 'Inverse SAR approach for localization of moving RFID tags', in 2013 IEEE International Conference on RFID, RFID 2013, 2013, pp. 104-109. 